\title{
Can experiment determine the stacking fault energy of metastable alloys?
}

\author{
Xun Sun ${ }^{\text {a,b }}$, Song Lu ${ }^{\text {b,* }}$, Ruiwen Xie ${ }^{\mathrm{b}}$, Xianghai An ${ }^{\mathrm{c}}$, Wei Li ${ }^{\mathrm{b}}$, Tianlong Zhang ${ }^{\mathrm{a}}$, Chuanxin Liang ${ }^{\mathrm{a}}$, \\ Xiangdong Ding a ${ }^{\text {, Yunzhi Wang }}{ }^{\mathrm{d}}$, Hualei Zhang ${ }^{\mathrm{a}, *}$, Levente Vitos ${ }^{\mathrm{b}, \mathrm{e}, \mathrm{f}}$ \\ a Frontier Institute of Science and Technology, State Key Laboratory for Mechanical Behavior of Materials, Xi'an Jiaotong University, Xi'an, 710049, China

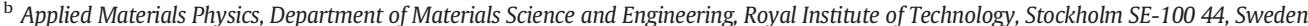 \\ ' School of Aerospace, Mechanical \&' Mechatronic Engineering, The University of Sydney, Sydney, NSW 2006, Australia \\ ${ }^{\mathrm{d}}$ Department of Materials Science and Engineering, The Ohio State University, 2041 College Road, Columbus, OH 43210, USA \\ e Division of Materials Theory, Department of Physics and Materials Science, Uppsala University, P.O. Box 516, SE-75120 Uppsala, Sweden \\ ${ }^{\mathrm{f}}$ Research Institute for Solid State Physics and Optics, Wigner Research Center for Physics, Budapest H-1525, P.O. Box 49, Hungary
}

\section{H I G H L I G H T S}

- The common models underlying experimental measurements of stacking fault energy fail in metastable alloys.

- Theoretical stacking fault energy correlates with the Gibbs free energy difference between the fcc and hcp phases.

- Ab initio calculated stacking fault energy correlates nicely with the prevailing deformation mechanism.

- Negative stacking fault energy plays critical role in understanding dislocation behaviors in metastable alloys.

\section{A R T I C L E I N F O}

\section{Article history:}

Received 20 October 2020

Received in revised form 6 December 2020

Accepted 7 December 2020

Available online 9 December 2020

\section{Keywords:}

Metastable alloy

Stacking fault energy

Twinning

Martensitic transformation

\section{G R A P H I C A L A B S T R A C T}

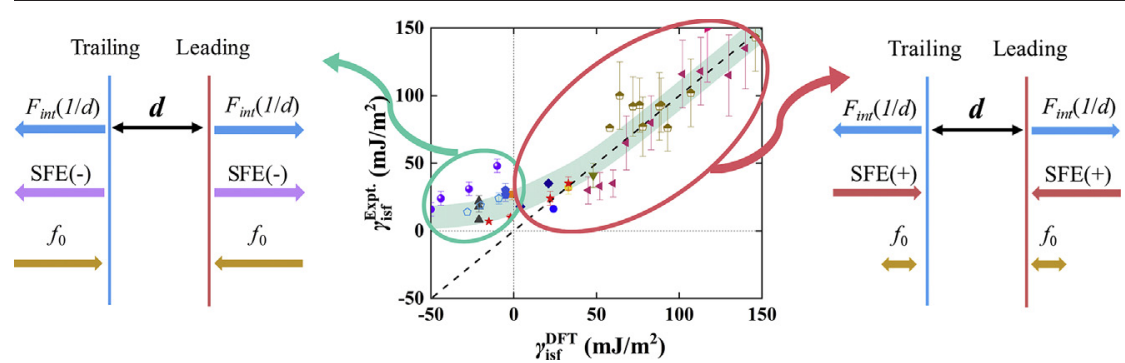

\begin{abstract}
A B S T R A C T
Stacking fault energy (SFE) plays an important role in deformation mechanisms and mechanical properties of face-centered cubic (fcc) metals and alloys. In many concentrated fcc alloys, the SFEs determined from density functional theory (DFT) calculations and experimental methods are found having opposite signs. Here, we show that the negative SFE by DFT reflects the thermodynamic instability of the fcc phase relative to the hexagonal close-packed one; while the experimentally determined SFEs are restricted to be positive by the models behind the indirect measurements. We argue that the common models underlying the experimental measurements of SFE fail in metastable alloys. In various concentrated solid solutions, we demonstrate that the SFEs obtained by DFT calculations correlate well with the primary deformation mechanisms observed experimentally, showing a better resolution than the experimentally measured SFEs. Furthermore, we believe that the negative SFE is important for understanding the abnormal behaviors of partial dislocations in metastable alloys under deformation. The present work advances the fundamental understanding of SFE and its relation to plastic deformations, and sheds light on future alloy design by physical metallurgy.
\end{abstract}

(c) 2020 The Author(s). Published by Elsevier Ltd. This is an open access article under the CC BY-NC-ND license (http://creativecommons.org/licenses/by-nc-nd/4.0/).

\footnotetext{
* Corresponding authors.

E-mail addresses: songlu@kth.se (S. Lu), hualei@xjtu.edu.cn (H. Zhang).
}

\section{Introduction}

Intrinsic stacking fault (ISF) in face-centered-cubic (fcc) materials is a type of two-dimensional defect which is usually created by the glide of a Shockley partial dislocation during deformation. Immediately, it is 
related to the nucleation of deformation twins and hexagonal closepacked (hcp) martensite which are generated by collective motions of partials. ISF is also the characteristic structure between the trailing and leading partials in a dissociated $a / 2\langle 110\rangle$ dislocation. From these aspects, the excess formation energy of ISF, i.e., the stacking fault energy (SFE), serves as a significant intrinsic material parameter affecting the dissociation of $a / 2<110>$ dislocations, twinning as well as martensitic transformation (MT). For example, in pure fcc metals, deformation twinning (DT) is often observed in Ag with a low SFE (measured value $16 \pm 2 \mathrm{~mJ} / \mathrm{m}^{2}$ [1]) but rarely in Al with a very high SFE (measured value $\sim 150 \pm 40 \mathrm{~mJ} / \mathrm{m}^{2}$ [2]). In austenitic Fe-Cr-Ni and Fe-Mn steels [3-9], an empirical relation between the prevalent deformation mode and the size of the measured SFE is established, showing that in addition to dislocation slips, low-SFE alloys $\left(<\sim 20 \mathrm{~mJ} / \mathrm{m}^{2}\right)$ prefer deformationinduced martensitic transformations (DIMTs) from fcc $(\gamma)$ to hcp $(\varepsilon)$ or to body-centered-tetragonal (bct, $\left.\alpha^{\prime}\right)$ phases, while medium-SFEs $\left(\sim 20-40 \mathrm{~mJ} / \mathrm{m}^{2}\right)$ prefer DT $[3,6,8,10,11]$. In materials with high SFEs, usually only dislocation slips can be observed under normal loading conditions [11]. The occurrence of DT or MT during plastic deformation provides important internal boundaries that interact with dislocations in distinct ways from grain boundaries [12], which enables the socalled dynamical Hall-Petch effect, maintaining the high workhardening rate and thereby delaying the onset of plastic instability and necking $[13,14]$. The resulting twinning-induced plasticity (TWIP) and transformation-induced plasticity (TRIP) are two important mechanisms responsible for the excellent plastic properties of high-Mn austenitic steels $[3,4,6-8]$ as well as some multicomponent solid solutions (also called medium- or high-entropy alloys, MEAs/HEAs) such as CrCoNi and CrMnFeCoNi [15-20]. Therefore, in the development of high-strength alloys, significant effort has been put to study factors that may affect the SFE, such as temperature [9,21-24], composition $[10,21,25]$ and short-range order (SRO) [26-31]; to understand the microscopic mechanisms underlying the role of SFE in DT, MT and dislocation planar slip [4,7,10,32-35]; as well as to quantitatively predict the critical twinning stress based on the SFE $[3,18,34,36-41]$ or the generalized SFE ( $\gamma$-surface) [42-44]. All these activities depend on the accurate determination of the SFE.

Nowadays, both experimental [45-47] and computational methods [42,48-52] are commonly applied to evaluate the SFEs. Particularly, ab initio methods based on density functional theory (DFT) calculations have been widely adopted to determine the SFE at $0 \mathrm{~K}$, as well as at elevated temperatures via considering contributions from thermal lattice expansion and electronic/phononic/magnetic excitations [23,53-56]. For pure fcc metals, a satisfying agreement between experimental and ab initio results has been reached for both the SFE values and the variation trends with respect to temperature $[48,53,54]$. However, the situation becomes more complicated for some alloys, especially the concentrated ones. For example, in $\mathrm{Cu}-\mathrm{Al}$ alloys ab initio calculations showed that the SFE decreases approximately linearly with increasing Al concentration and turns to be negative at 10 at.\% $\mathrm{Al}[48,57]$; whereas the experimentally determined values first decrease and then become constant at $\sim 5 \mathrm{~mJ} / \mathrm{m}^{2}$ for Al concentration higher than 10 at.\% [22]. Similar observation can be made for $\mathrm{Ni}-\mathrm{Co}$ alloys with respect to Co concentration $[21,22,58-60]$. It seems that the temperature effect cannot systematically improve the agreement between the theoretical calculations and experimental measurements of SFEs in pure metals (or dilute alloys) and concentrated alloys. Recently, in the development of HEAs, the experimental and theoretical SFEs often have opposite signs (see Table 1), which cannot be ascribed solely to the inappropriate treatment of the temperature effect in ab initio calculations [13,15,17,26,61-68]. Further efforts to resolve the problem including the consideration of SRO or local chemical variations have been made. For instance, atomistic studies demonstrated that the SFE depends on the local chemical composition as well as chemical and magnetic SROs [26-31]. Ding et al. [26] reported that the calculated mean SFE of CrCoNi MEA increases markedly from $-42.9 \mathrm{~mJ} / \mathrm{m}^{2}$ at the fully random state (estimated through averaging the SFEs of 108 configurations with a broad distribution from -140 to $65 \mathrm{~mJ} / \mathrm{m}^{2}$ ) to $\sim 30 \mathrm{~mJ} / \mathrm{m}^{2}$ with increasing the degree of SRO, in comparison to the experimental values, $22 \pm 4$ [61] and $18 \pm 4 \mathrm{~mJ} / \mathrm{m}^{2}$ [17]. Thereby, the authors ascribed the discrepancy in the SFEs in these multicomponent alloys to the SROs. Li et al. [28] further argued that the wide variety of local chemical ordering produces a wide range of generalized stacking fault energies, therefore, increasing the ruggedness of the energy landscape for dislocation activities and influencing the selection of dislocation pathways in slip, faulting and twinning. Being aware of the difficulties of experimental methods in characterizing SROs, recent experiments have found that in the homogenized $\mathrm{CrCoNi}$ specimens, SRO (or local chemical variation) is however very weak, if not absent $[62,69,70]$; only by aging the homogenized CrCoNi MEA at $1000{ }^{\circ} \mathrm{C}$ for $120 \mathrm{~h}$ followed by furnace cooling, Zhang et al. [62] reported observation of SRO domains of $\sim 1 \mathrm{~nm}$ and that the measured SFE based on partial separation distance increases from $8.18 \pm 1.43 \mathrm{~mJ} / \mathrm{m}^{2}$ for the water-quenched sample to $23.33 \pm$ $4.31 \mathrm{~mJ} / \mathrm{m}^{2}$ for the annealed one. Therefore, the discrepancy in the theoretical and experimental SFEs is not solved in CrCoNi MEA, neither in other HEAs. Additionally, one may notice the different experimental SFE values for the homogenized CrCoNi MEA in different Refs. $[17,61,62]$, despite that they are all based on transmission electron microscopy (TEM) measurement of stacking fault width (SFW), which was also ascribed to the inherent local chemical variations in these alloys [29]. Smith et al. [29] found large variations in dislocation dissociation distances through analyzing the measured results for 30 different $a$ / $2<110>\{111\} 60^{\circ}$ mixed dislocations in the CrMnFeCoNi HEA. The average separation distance was $4.82 \mathrm{~nm}(\sim 5.5 \mathrm{~nm}$ [13]) but with a large range of variation $( \pm 3.4 \mathrm{~nm})$, an order of magnitude higher than the normal variation $( \pm 0.45 \mathrm{~nm})$ in pure fcc metals [29]. No evidence of alloying element segregation or ordering was found in the alloy [70], but the large variations in dislocation separations were still ascribed to the local chemical inhomogeneity. It is however not clear how the local composition inhomogeneity or SROs, if exist, in the length scales of $\sim 1 \mathrm{~nm}$ or less $[26,62,69]$ affect the behaviors of dislocations with significantly longer lengths and larger separation widths in the processes of faulting or twinning.

$\mathrm{Ab}$ initio calculations for the thermodynamic parameters like the SFE as a function of composition in alloys are efficient in screening for proper candidates before conducting experiments. To facilitate future design of alloys with vast composition space, it is important to better understand the ab initio and experimental methodologies for the SFE determination, especially their limitations. Here, we aim at answering the following questions: can experimental methods determine the SFEs of metastable alloys and what is the relationship between the measured and the ab initio SFEs in these systems? In the present work, we focus on the metastable alloys defined by a higher Gibbs free energy of the fcc phase than that of the hcp one $\left(\Delta G^{\gamma \rightarrow \varepsilon}<0\right)$, which is the most relevant case for the discussions of the deformation mechanisms in fcc solid solutions. Although metastability may also refer to the thermodynamic states of other phases such as decomposed phases and precipitates, their impact is not discussed here.

The rest of the paper is organized as following. In Section 2, we give a brief introduction of the adopted theoretical approaches for the SFE calculations, more details of the methodologies can be found in our previous publications [56,71]. Results and discussion are given in Section 3. We start with analyzing the limitations of current experimental approaches for measuring the SFE in subsection 3.1. The correlations between the calculated/experimental SFEs and phase stability are presented in subsection 3.2. In subsection 3.3 we analyze the correlation between the theoretical SFEs and the observed deformation mechanisms. Further implications of the present findings are discussed in subsection 3.4. Conclusions are drawn in Section 4. 
Table 1

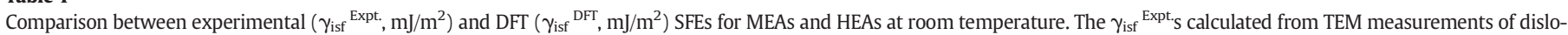

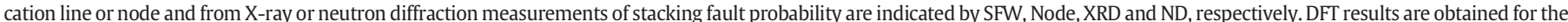

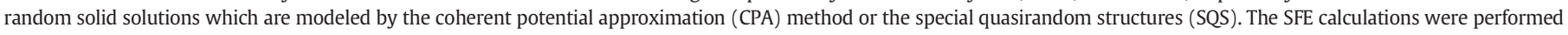

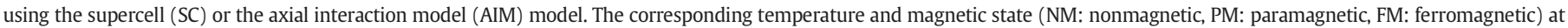
which the calculations are performed are also indicated. The used lattice constants ( $a$ in $\AA$ ) for the SFE calculations are given.

\begin{tabular}{|c|c|c|c|c|c|c|}
\hline Alloys (at. \%) & $\gamma_{\text {isf }}$ Expt. & $a(\AA)$ & Experiment & $\gamma_{\text {isf }}$ DFT & $a(\AA)$ & Calculation \\
\hline \multirow[t]{10}{*}{$\mathrm{CrCoNi}$} & $18 \pm 4[17]$ & 3.529 & SFW & -21 & $3.567[61]$ & CPA, SC, 300 K, PM \\
\hline & $22 \pm 4[61]$ & 3.567 & SFW & -29 & 3.526 & CPA, SC, 0 K, PM \\
\hline & $8.18 \pm 1.43[62]$ & 3.564 & SFW & $-43[26]$ & 3.526 & SQS, SC, 0 K, FM \\
\hline & & & & $-24[66]$ & 3.516 & SQS, SC, $0 \mathrm{~K}, \mathrm{NM}$ \\
\hline & & & & $-62[67]$ & 3.532 & SQS, SC, 0 K, FM \\
\hline & & & & $-38[68]$ & -- & SQS, SC, 0 K, FM \\
\hline & & & & $-25[67]$ & -- & SQS, AIM, 0 K, FM \\
\hline & & & & $-5[67]$ & - & SQS, AIM, 300 K, FM \\
\hline & & & & $-58[68]$ & - & SQS, AIM, 0 K, FM \\
\hline & & & & $-51[68]$ & -- & SQS, AIM, 300 K, FM \\
\hline \multirow[t]{3}{*}{ CrFeCoNi } & $27 \pm 4[17]$ & 3.565 & SFW & -1 & $3.577[78]$ & CPA, SC, 300 K, PM \\
\hline & $\sim 20-25$ [63] & 3.575 & XRD & -27 & 3.526 & $\mathrm{CPA}, \mathrm{SC}, 0 \mathrm{~K}, \mathrm{PM}$ \\
\hline & $32.5[15]$ & 3.604 & ND & $-23[67]$ & 3.540 & SQS, SC, 0 K, FM \\
\hline \multirow[t]{8}{*}{ CrMnFeCoNi } & $30 \pm 5[13]$ & 3.606 & SFW & -5 & 3.594 [79] & CPA, SC, 300 K, PM \\
\hline & $26.5 \pm 4.5[17]$ & 3.576 & SFW & -73 & 3.526 & CPA, SC, 0 K, PM \\
\hline & $\sim 20-25$ [63] & 3.597 & XRD & $-54[67]$ & 3.541 & SQS, SC, 0 K, FM \\
\hline & & & & $-31[68]$ & - & SQS, SC, $0 \mathrm{~K}, \mathrm{FM}$ \\
\hline & & & & $-45[68]$ & - & SQS, AIM, 0 K, FM \\
\hline & & & & $-28[68]$ & - & SQS, AIM, 300 K, FM \\
\hline & & & & $-93[82]$ & - & CPA, AIM, 0 K, PM \\
\hline & & & & $-14[82]$ & -- & CPA, AIM, $300 \mathrm{~K}, \mathrm{PM}$ \\
\hline $\mathrm{Cr}_{20} \mathrm{Mn}_{20} \mathrm{Fe}_{20} \mathrm{Co}_{23} \mathrm{Ni}_{17}$ & $24 \pm 4[64]$ & 3.598 & SFW & -9 & $3.598[64]$ & CPA, $300 \mathrm{~K}, \mathrm{PM}$ \\
\hline $\mathrm{Cr}_{20} \mathrm{Mn}_{20} \mathrm{Fe}_{20} \mathrm{Co}_{27} \mathrm{Ni}_{13}$ & $19 \pm 3[64]$ & 3.595 & SFW & -20 & $3.595[64]$ & CPA, $300 \mathrm{~K}, \mathrm{PM}$ \\
\hline $\mathrm{Cr}_{20} \mathrm{Mn}_{20} \mathrm{Fe}_{20} \mathrm{CO}_{30} \mathrm{Ni}_{10}$ & $\sim 14$ [64] & 3.593 & SFW & -28 & $3.593[64]$ & CPA, $300 \mathrm{~K}, \mathrm{PM}$ \\
\hline $\mathrm{Fe}_{40} \mathrm{Mn}_{40} \mathrm{Co}_{10} \mathrm{Cr}_{10}$ & $13 \pm 4[65]$ & 3.61 & Node & -3 & $3.621[114]$ & CPA, $300 \mathrm{~K}, \mathrm{PM}$ \\
\hline
\end{tabular}

\section{Methodology}

All DFT calculations were performed using the exact muffin-tin orbitals (EMTO) method [72] in combination with the single-site coherent potential approximation (CPA) [73]. The computational package can be found on the EMTO website [74]. Both the self-consistent calculations and total energies were calculated within the PerdewBurke-Ernzerholf (PBE) exchange-correlation functional [75]. The scalar-relativistic approximation and soft-core scheme were adopted to solve the one-electron Kohn-Sham equations. The Green's function was calculated self-consistently for 16 complex energy points. We employed an $l$-cutoff of 8 in the one-center expansion of the fullcharge density. The paramagnetic (PM) state was described by the disordered local magnetic moment (DLM) model [76,77]. The $9 \times 18 \times 2 k$-point mesh was used for all supercell calculations after careful tests. A careful assessment of the present method for the determination of the SFE in fcc metals and alloys can be found in Ref. [48]. The SFEs were calculated using supercells formed by 12 closepacked $\{111\}$ layers. The intrinsic stacking fault $(A B C \mid B C A B C)$ is formed when a layer of atoms is removed from a perfect fcc sequence $(\mathrm{ABCABCABC})[47]$. The SFE was computed as SFE $=\left(F^{\text {fault }}-F^{0}\right) / A$, where $F^{\text {fault }}$ and $F^{0}$ are the free energies with and without a stacking fault, respectively, and $A$ is the stacking fault area. In the previous works $[42,48,56]$, this supercell method was successfully used to calculate the SFEs of pure metals and solid solutions. The free energies were approximated as $F=E-T S_{\mathrm{mag}}$, where $T$ is the temperature, and $E$ is the internal energy. The magnetic entropy $S_{m a g}=$ $k_{B} \sum_{i=1}^{n} c_{i} \ln \left(1+\mu_{i}\right)$ was calculated within the mean-field approximation, where $k_{\mathrm{B}}$ is the Boltzmann constant, $n$ is the total number of elements, $c_{i}$ and $\mu_{i}$ are the concentration and local magnetic moment for the $i$ th alloying element, respectively. To partly include the room temperature (RT) effect on the SFE, we used the experimental lattice parameters measured $[61,64,78,79]$ at RT or obtained by a regression formula [78]. Electronic and explicitly anharmonic phonon contributions were estimated to be small at RT and they were neglected
[80], which however does not affect the discussions in the present work. For more details regarding the treatment of the temperature effect on the SFE calculation and the corresponding errors, readers are referred to Refs. [53, 54, 81, 82].

\section{Results and discussion}

\subsection{Limitations of experimental methods for SFE determination}

SFE is usually considered as an experimentally accessible parameter by means of TEM, X-ray or neutron diffractions. In the following, we discuss the limitations of the experimental methods for determining the SFE, especially in metastable alloys. Methods involving TEM measure the distance $(d)$ between the two $a / 6\langle 112\rangle$ partial dislocations in a dissociated $a / 2<110>$ dislocation [45] or the radii describing the size of the dislocation node [46], and then one connects these parameters to the SFE. In order to minimize the interaction between dislocations, the partial separation width $d$ is usually measured on an isolated straight dislocation line at its equilibrium state, then the SFE is calculated according to

$\gamma_{\text {isf }}^{\text {Expt. }}=f\left(b_{p}, \mu, \nu, \beta\right) / d$,

with $f\left(b_{p}, \mu, \nu, \beta\right)=\frac{\mu b_{p}{ }^{2}}{8 \pi} \frac{2-v}{1-v}\left(1-\frac{2 v \cos 2 \beta}{2-v}\right)$,

where $\mu$ is the shear modulus on the $\{111\}$ close-packed planes, $b_{\mathrm{p}}$ is the Burgers vector of partial dislocation, $v$ is the Poisson ratio, $\beta$ is the angle between Burgers vector $b$ and dislocation line direction. It is important to notice that the above equation is established based on the presumed balance between two forces acting on a partial dislocation: namely the repulsive force ( $F_{\text {int }}$ ) (per unit length of dislocation line) due to the elastic interaction between the two partials (leading and trailing) and the attractive force due to the excess energy cost to create a stacking fault (SF), i.e., the SFE (Fig. 1(a)). The right hand term in 

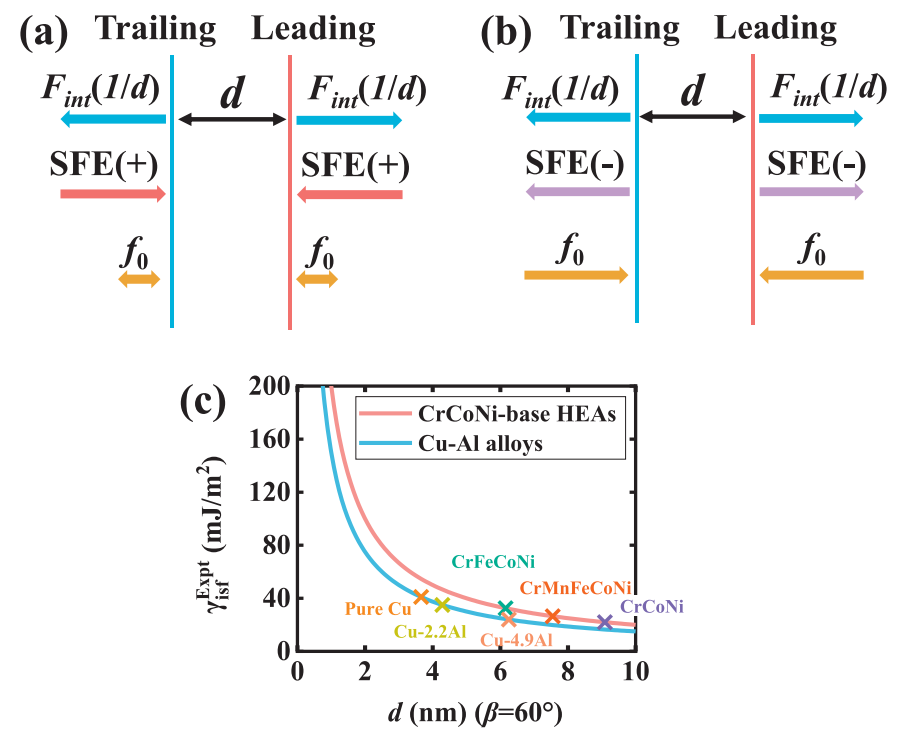

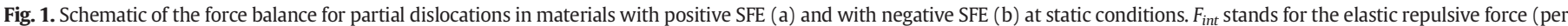

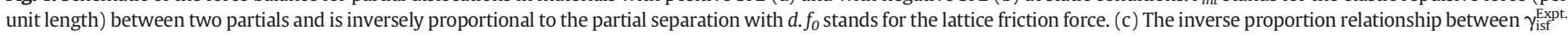

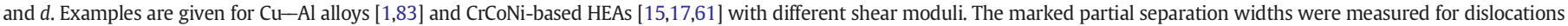
with $\beta=60^{\circ}$.

Eq. (1) corresponds to the repulsive force calculated according to the

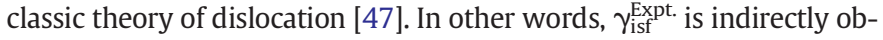
tained from the elastic repulsive force through the measured $d$. Several critical points can then be drawn from Eq. (1):

(1) For dislocations of the same character $(\beta)$, the $F_{\text {int }}$ and the socalculated $\gamma_{\text {isf }}^{\text {Expt. }}$ are inversely proportional to $d$, which are always positive and approach to zero as $d$ increases, see Fig. 1(c).

(2) In the case of small partial separation (corresponding to large SFE), a small scatter in $d$ measurement can cause a large deviation in $\gamma_{\text {isf }}^{\text {Ext. }}$, thereby, the TEM method is usually thought to be proper for measuring small SFEs to avoid the large uncertainty $[1,17,61,64]$.

(3) In the case of large separation, the $\gamma_{\text {isf }}^{\text {Expt. }}$ quickly decreases toward a small positive value (e.g., $<20 \mathrm{~mJ} / \mathrm{m}^{2}$ ) which barely changes with increasing $d$ within the experimental error bar; in other words, $\gamma_{\text {isf }}^{\text {Ext. }}$ gradually loses the resolution in identifying the dislocation separation. This observation is important for understanding the temperature effect on the experimental SFE in various alloys. For example, for alloys with positive temperature dependence of the SFE, the measured SFE appears almost constant at low temperatures [84-87].

(4) For fcc metals and alloys, $\nu$ is usually around $0.3[13,17]$. The proportional factor $\left(f\left(b_{p}, \mu, \nu, \beta\right)\right)$ mainly depends on the corresponding shear moduli of different materials. For the same partial separation width, the $\gamma_{\text {isf }}^{\text {Expt. }}$ can differ significantly in alloys with different shear moduli, which explains why the critical $\gamma_{\text {isf }}^{\text {Expt. }} s$ separating different deformation mechanisms differ significantly in various alloys [11]. In other words, the same $\gamma_{\text {isf }}^{\text {Expt. }}$ value in different alloy systems may correspond to different partial separation widths and indicates different states of thermodynamic stability of the fcc phase.

Most critically, the true SFE has always to be positive to potentially allow Eq. (1) to hold, neglecting any other factors (e.g., the lattice friction force $[34,88]$ ) that can participate in establishing the equilibrium stacking fault ribbon. On the other hand, the sign of the SFE can to some extend indicate the thermodynamic stability of the fcc phase as discussed in the following. First, by definition, the SFE is the excess formation energy of the intrinsic stacking fault relative to the energy state of the fcc structure. From the structural point of view, the stacking fault can be seen as a two-layer of hcp embryo embedded in the fcc matrix, thereby the SFE can be expressed as [5]

$\mathrm{SFE}=\frac{2\left(\Delta G^{\gamma \rightarrow \varepsilon}+E^{s t r .}\right)}{A}+2 \sigma^{\varepsilon / \gamma}$,

where $\Delta G^{\gamma \rightarrow \varepsilon}$ is the Gibbs energy difference (per atom) between the hcp and fcc phases, $E^{\text {str. }}$ is the strain energy contribution, $A$ is the stacking fault area and $\sigma^{\varepsilon / \gamma}$ is the coherent interfacial energy between fcc and the two-layer hcp embryo [5]. $E^{\text {str. }}$ is a small positive term giving a contribution of $\sim 1-4 \mathrm{~mJ} / \mathrm{m}^{2}[5,89]$. The interfacial energy for the coherent $\mathrm{fcc}\{111\} / \mathrm{hcp}(0001)$ interface depends on the thickness of the hcp phase; and therefore $\sigma^{\varepsilon / \gamma}$ for the two-layer hcp embryo usually differs from the value obtained between bulk fcc and bulk hcp [90]. Previous ab initio studies showed that $\sigma^{\varepsilon / \gamma}$ is in the range of $\pm 9 \mathrm{~mJ} / \mathrm{m}^{2}$ [90]. When the Gibbs energy difference is small, $\sigma^{\varepsilon / \gamma}$ and $E^{\text {str. }}$ together determine the sign of the SFE. However, when the hcp structure is much more stable than the fcc one (typically, $\Delta G^{\gamma \rightarrow \varepsilon} \lesssim-0.2 \mathrm{mRy} / \mathrm{atom}$ ), the SFE of the metastable fcc phase as computed from Eq. (2) becomes negative. Obviously in this case one cannot use Eq. (1) to determine the SFE because both the SFE and the elastic repulsive force point to the same direction and the force balance condition between these two terms breaks down (Fig. 1(b)). We argue that in the absence of external shear stress, it is the lattice friction force on the partial dislocations which can ensure the force balance condition and thus lead to finite partial separations in metastable systems $[34,88]$. Therefore, for metastable fcc alloys, Eq. (1) breaks down and cannot be adopted for deriving the experimental SFE. We note that in the positive-SFE cases like pure fcc metals, the lattice friction force/stress should also exist, but it does not play a dramatic role as in the case of negative SFE, which will be further discussed in the following sections.

Similar arguments apply for the SFE measurement by using the dislocation node method $[46,91]$. Furthermore, measuring the SFE by using $\mathrm{X}$-ray or neutron diffraction suffers from similar restrictions since the support for the measurement is also based on the above-discussed force balance assumption to derive the SFE [92]. There, the partial separation width $d$ is replaced by the stacking fault probability through $\alpha=$ $\frac{\rho d a_{0}}{\sqrt{3}}$, where $a_{0}$ is the lattice constant, and $\rho=K_{111}\left\langle\epsilon_{50}^{2}\right\rangle_{111} / b^{2}, K_{111}$ is a 
constant and $\left\langle\epsilon_{50}^{2}\right\rangle_{111}$ is the rms microstrain in the [111] direction averaged over a distance of $50 \AA . \alpha$ is then measured from the shift in the position of the diffraction lines [93]. Consequently, $\gamma_{\text {isf }}^{\text {Expt. }}$ obtained by those experimental methods will all depart from the thermodynamic stability of the fcc phase (expressed via $2 \Delta G^{\gamma \rightarrow \varepsilon} / A$ ) in metastable systems.

\subsection{Correlations between ab initio SFE, $\Delta G^{\gamma \rightarrow \varepsilon}$, and experimental SFE}

In Fig. 2(a), we compare $\gamma_{\text {isf }}^{\mathrm{DFT}}$ and $2 \Delta G^{\gamma \rightarrow \varepsilon} / A$, both calculated by DFT method for various fcc metals and alloys. The two quantities correlate nicely with each other, as described in Eq. (2), even when SROs are present (points connected by lines) [26]. The observation is consistent with the previous studies $[26,48,97,99]$. The strain energy contribution is not included in the calculations, therefore, the deviation between the two quantities gives the interfacial energy term $\left(2 \sigma^{\varepsilon / \gamma}\right) . \sigma^{\varepsilon / \gamma}$ is estimated in the range of $-9 \sim 8 \mathrm{~mJ} / \mathrm{m}^{2}$ for the studied metals and alloys shown in Fig. 2(a), which is in agreement with the previous values calculated by DFT methods [90].

In Fig. 2(b), we compare the SFEs obtained by DFT ( $\left.\gamma_{\text {isf }}^{\mathrm{DFT}}\right)$ and by experiments ( $\left.\gamma_{\text {isf }}^{\text {Ext. }}\right)$ for various thermodynamically stable and unstable fcc metals and alloys. One can observe that for fcc elemental metals ( $\mathrm{Ag}, \mathrm{Cu}, \mathrm{Au}$ and $\mathrm{Al}$, etc.) and the stable fcc alloys (e.g., Pd-Ag and PdCu alloys) with $\Delta G^{\gamma \rightarrow \varepsilon}>0$ (Fig. 2(a)), DFT and experiments lead to consistent results, implying that the force balance condition assumed in Eq. (1) is indeed satisfied (Fig. 1(a)), in other words, the lattice friction forces are negligible in these cases. We may estimate the magnitude of the lattice friction force from the Peierls stress $\left(\tau_{P}\right)$ in pure fcc metals. Experimentally, the upper limit of Peierls stress is usually estimated from the critical resolved stress for slip extrapolated to $0 \mathrm{~K}$, which is less than $\sim 10 \mathrm{MPa}$ for pure fcc metals $\left(\tau_{P} / \mu \sim 10^{-4}-10^{-5}\right.$, $\mu$ is the shear modulus, see Refs. $[100,101]$ and Refs. therein). Then the lattice friction force on full dislocations can be calculated as $\tau_{P} \cdot b \approx 3 \mathrm{~mJ} / \mathrm{m}^{2}$ (PeachKohler force [47]), which sets the upper limits of the friction forces on the partial dislocations. One can see that the friction force is negligible, which is at the same order of magnitude of the errors in the experimental/DFT SFEs, and falls in the range of the discrepancy between $\gamma_{\text {isf }}^{\mathrm{DFT}}$ and $\gamma_{\text {isf }}^{\text {Expt. }}$ for pure fcc metals.

But for those metastable fcc alloys $\left(\Delta G^{\gamma \rightarrow \varepsilon}<0\right)$ including binary Co$\mathrm{Ni}$ alloys, $\mathrm{Cu}-\mathrm{Al}$ alloys with high $\mathrm{Al}$ concentrations, ternary $\mathrm{Cr}-\mathrm{Co}-\mathrm{Ni}$ MEAs and quinary Cr-Mn-Fe-Co-Ni HEAs, the theoretical and experimental values deviate notably from each other, which is primarily ascribed to the reasons discussed in Section 3.1. The uncertainties in DFT calculations are discussed in the following sections, but they cannot be responsible for the opposite signs of $\gamma_{\text {isf }}^{\mathrm{DFF}}$ and $\gamma_{\text {isf }}^{\text {Expt. in these metastable }}$ alloys, especially when $\Delta G^{\gamma \rightarrow \varepsilon}$ is very negative, e.g., in fcc Co and high-Co alloys $[60,102]$. Similar conclusion can be reached when comparing the experimental SFE and the Gibbs energy difference $2 \Delta G^{\gamma \rightarrow \varepsilon} / A$ obtained by CALPHAD calculations (Fig. 2(c)).

Since pairs of partial dislocations are still observed in these metastable alloys, one expects the presence of large friction forces preventing the separation of full dislocations into very wide SFs or the spontaneous formation of hcp martensite. There is experimental evidence of large lattice friction existing in these alloys. For example, the Peierls stresses for $\mathrm{CrCoNi}$ and $\mathrm{CrMnFeCoNi}$ were estimated to be $\sim 160 \mathrm{MPa}$ and $\sim 138 \mathrm{MPa}$, respectively, approximately one order of magnitude higher than that of $\mathrm{Ni}(\sim 15 \mathrm{MPa})$ [103]. Yoshida et al. [104] measured the friction stress term $\left(\sigma_{0}\right)$ in the Hall-Petch relation, $\sigma_{Y S}=$ $\sigma_{0}+k \lambda^{1 / 2}$, where $\sigma_{Y S}$ is the yield strength, $k$ is Hall-Petch slope and $\lambda$ is the mean grain size. They showed that the friction stress is significantly larger for $\mathrm{CrCrNi}$ (218 MPa) and CrMnFeCoNi (125 MPa) than those for pure $\mathrm{Al}$ (4 MPa) and $\mathrm{Ni}$ (14 MPa). Particularly, Lee et al. [70] measured the critical resolved shear stress acting on moving
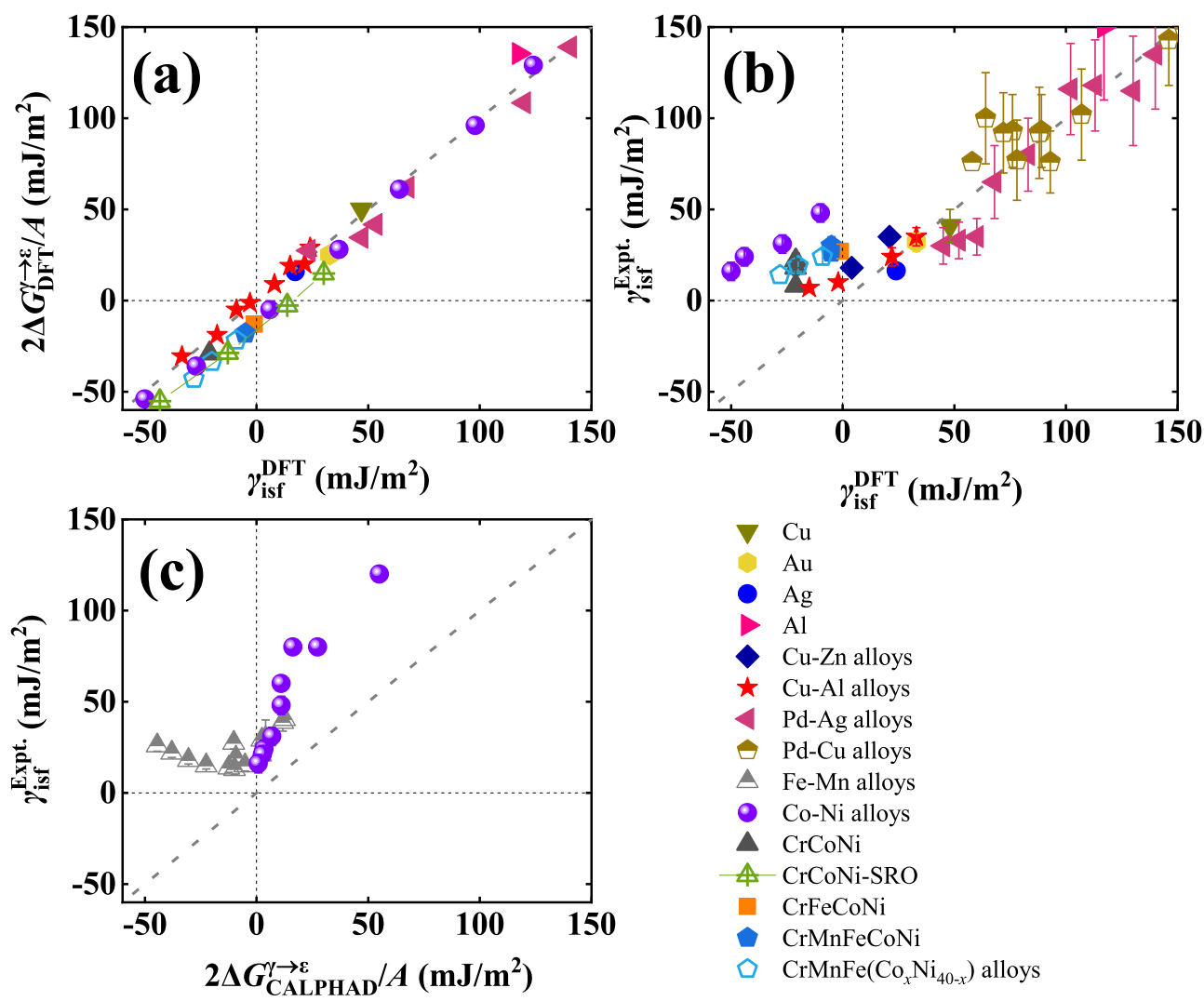

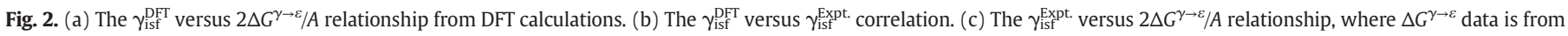

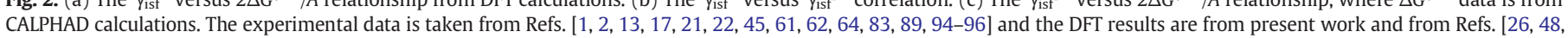
$90,97]$. CALPHAD results for Fe-Mn alloys are from Ref. [89], and those for Co-Ni alloys are calculated using TTNI8 database [98]. 
dislocations according to the curvature of the dislocations during deformation of $\mathrm{Cr}_{20} \mathrm{Mn}_{12} \mathrm{Fe}_{24} \mathrm{Co}_{24} \mathrm{Ni}_{20}$. The average shear stresses on two representative dislocations are found to be $525 \pm 65 \mathrm{MPa}$ and $465 \pm$ $62 \mathrm{MPa}$, which are significant values. They also showed that some dislocations are still curved after the release of the external stress due to the high lattice friction which hinders dislocations from returning to straight line shape. The shear stress in the static dislocations is measured to be $144 \pm 56 \mathrm{MPa}$. There are three possible contributions to the resolved shear stress required for dislocation moving: (1) dislocation pinning effect at the surface of TEM samples; (2) thin foil effects and (3) lattice friction. Both static and moving dislocations are affected similarly by the first two factors. Therefore, the significant increase in the shear stress from static dislocations $(144 \pm 56 \mathrm{MPa})$ to gliding dislocations ( $525 \pm 65 \mathrm{MPa}$ or $465 \pm 62 \mathrm{MPa}$ ) may be mainly ascribed to the lattice friction, which is larger than $\sim 300 \mathrm{MPa}$. One may expect similar level of lattice friction in other MEAs and HEAs, e.g., in the equiatomic $\mathrm{CrMnFeCoNi} \mathrm{HEA}$. Based on friction stress of $300 \mathrm{MPa}$, the friction force on full dislocations calculated according to the Peach-Kohler force $(\tau \cdot b)$ is about $\sim 80 \mathrm{~mJ} / \mathrm{m}^{2}$, indicating that the large lattice friction can provide enough resistant force to balance the elastic repulsive force $\left(30 \pm 5 \mathrm{~mJ} / \mathrm{m}^{2}\right.$ [13], $26.5 \pm 4.5 \mathrm{~mJ} / \mathrm{m}^{2}$ [17]) and the negative SFE $\left(-5 \mathrm{~mJ} / \mathrm{m}^{2}\right)$, which is consistent with our above discussions. The large lattice friction in these alloys is related to the significantly roughened Peierls potential surface due to e.g., local lattice distortions or complexed chemical/magnetic configurations $[28,104,105]$.

In Table 1, we summarize the calculated SFE values by various DFT methods for the CrCoNi-based MEAs and HEAs as well as the $\mathrm{Fe}_{40} \mathrm{Mn}_{40} \mathrm{Co}_{10} \mathrm{Cr}_{10}$ TWIP HEA [26,66-68], whose signs are all in stark contrast with the experimental ones [13,15,17,61-65]. In particular, for the CrCoNi MEA which has been extensively studied by various experimental and theoretical methods due to its excellent mechanical performance, all DFT calculations yield negative $\gamma_{\text {isf }}^{\mathrm{DFT}}$ and $\Delta G^{\gamma \rightarrow \varepsilon}$ for the random solid solution state at both room and cryogenic temperatures [66-68]. $\gamma_{\mathrm{isf}}^{\mathrm{DFT}}$ and $\Delta G^{\gamma \rightarrow \varepsilon}$ are in nice correlation with each other, even when short-range orders are considered [26], which follows the consideration from the structural point of view. Despite the fact that variations in chemical or magnetic SROs may significantly alter the calculated SFE $[26,28,30]$, the positive $\gamma_{\text {isf }}^{\text {Expt. }}$ comes inherently from the experimental methodologies and does not necessarily relate to the state of SROs in the materials [106]. Note that the experimental SFE is calculated by fitting Eq. (1) to many measured SFW $(d)$ values. Locally, each $d$ may be affected by the local chemical environment, but the obtained experimental SFE will be an averaged value anyway. Furthermore, recent experiments showed that the element distribution in the homogenized CrCoNi MEA is in fact highly random and homogeneous $[62,69,70]$. The metastability of the random CrCoNi MEA, as well CrMnFeCoNi HEA, is evidenced by the high pressure experiments showing that the $\mathrm{fcc} \rightarrow$ hcp phase transformation occurs during pressing at ambient temperature [107-109]. The pressure-induced hcp martensite was shown stable at ambient conditions after the release of the high pressure and transferring to the high temperature fcc phase after heating [108]. Additionally, Chen et al. found the coexistence of the hcp and fcc phases in the epitaxial film where the hcp phase can reach very high fraction [110]. As discussed above, it is likely the large lattice friction stress $[103,104]$ that prevents the spontaneous nucleation of hcp martensite in these alloys at room temperature, despite that the hcp structure is energetically more stable than the fcc one.

Before we end this section, in the following we discuss more details on the effect of temperature on the SFE in ab initio calculations, which is usually separated into several contributions including thermal lattice expansion, explicit phonon excitation, electronic excitation, and magnetic excitation. When the SFE is estimated according to $\gamma=2 \Delta G^{\gamma \rightarrow \varepsilon}$ / $A$, the temperature effect of the SFE may be assessed through the calculations of $G^{\gamma}$ and $G^{\varepsilon}$ with quasi-harmonic approximation. For example, Niu et al. [68] found that the calculated SFE of CrCoNi increases from $-58 \mathrm{~mJ} / \mathrm{m}^{2}$ at $0 \mathrm{~K}$ to $-51 \mathrm{~mJ} / \mathrm{m}^{2}$ at $300 \mathrm{~K}$. Zhao et al. [67] reported the SFE of $-5 \mathrm{~mJ} / \mathrm{m}^{2}$ at room temperature and $-25 \mathrm{~mJ} / \mathrm{m}^{2}$ at $0 \mathrm{~K}$ for $\mathrm{CrCoNi}$. Notice that in these studies, magnetic excitation was neglected. Contribution from electronic excitation is usually very small and negligible $[82,102]$. The total vibrational contribution is largely due to the thermal lattice expansion and the explicit phonon contribution is small. Therefore, the significant part of temperature effect on the SFE may be obtained by considering thermal lattice expansion, as shown in Refs. [23, 111], which significantly simplifies the calculations. Using Debye-Grüneisen model, Ma et al. calculated the vibrational entropy for $\mathrm{CrMnFeCoNi}$ [82] and they showed that vibrational entropy difference between fcc and hcp structures is up to $\sim 0.5 k_{\mathrm{B}}$ which does not vary significantly with increasing temperature. They also showed that at the paramagnetic state $\Delta G^{\gamma \rightarrow \varepsilon}$ is negative up to $340 \mathrm{~K}$, which is consistent with the present result and the result obtained by Niu et al. [68]. Similarly, in paramagnetic fcc Fe and Fe-Mn steels, the temperature effect on the SFE is also largely due to thermal lattice expansion. For example, Razumovskiy et al. [112] showed that electronic contribution is relatively small and the magnetic entropy contribution is almost constant at elevated temperatures. The overall temperature dependence of the SFE is mainly from thermal lattice expansion. Similar results were reported by Lu et al. [113].

\subsection{Correlation between ab initio SFE and deformation mechanism}

As we introduced in the Introduction, the occurrence of DT or $\gamma \rightarrow \varepsilon$ DIMT is usually rationalized with the sizes of the experimental SFEs. Being aware of the limitations of the experimental methods in determining the SFE, instead, here we may establish the relationship between the ab initio SFE and the deformation mode. In Fig. 3, we demonstrate the correlation between $\gamma_{\mathrm{isf}}^{\mathrm{DFT}}$ and the observed prevalent deformation mechanisms [61,64,115-117] in a group of MEAs and HEAs. We emphasize here that despite of the significant complexity of the compositions of these multicomponent alloys, the calculated $\gamma_{\text {isf }}^{\mathrm{DFT}}$ acts as a reliable indicator for the activation of the primary deformation mechanisms. Compared to the experimental SFEs (usually available for TWIP, but not for TRIP alloys due to the fact that a large amount of wide SFs exist already at very small strains, which prevents meaningful measurements of partial separation width), the ab initio SFEs have a better resolution in indicating the twinnability or the tendency to DIMT. It becomes even more striking if one includes the results of binary alloys (e.g., $\mathrm{Cu}-\mathrm{Al}$ ) from Fig. 2. For all the studied alloys, the $\gamma_{\mathrm{isf}}^{\mathrm{DFT}} \mathrm{S}$ are negative at room temperature, suggesting the metastable nature of these alloys. There seems a critical value or transition region of $\gamma_{\mathrm{isf}}^{\mathrm{DFT}}$ at around $-20 \mathrm{~mJ} / \mathrm{m}^{2}$ corresponding approximately to the case of $\mathrm{CrCoNi}$ MEA, that separates the observed primary deformation mechanisms and the operation of the TWIP and TRIP effects. The microscopic mechanism for this observation is discussed in a separate work [118]. We should mention here that although DT is the dominated mechanism in the CrCoNi MEA, small amounts of hcp martensite has indeed been observed during deformation [119-121]. Similarly for the $\mathrm{Cr}_{20} \mathrm{Mn}_{20} \mathrm{Fe}_{20} \mathrm{Co}_{30} \mathrm{Ni}_{10}$ HEA in the TRIP region, whose $\gamma_{\text {isf }}^{\mathrm{DFT}}$ is $-28 \mathrm{~mJ} /$ $\mathrm{m}^{2}$, locating close to the transition zone, it was observed that SFs and DT occur at small strains and hcp MT becomes dominant at large strains [64]. Thus, these MEAs and HEAs with the $\gamma_{\text {isf }}^{\mathrm{DFT}}$ in the transition zone are likely to show joint TWIP+TRIP effects, together with simultaneously enhanced synergy of strength and ductility $[17,61,64,115]$. Accordingly, for the $\mathrm{Cr}_{25} \mathrm{Fe}_{25} \mathrm{CO}_{35} \mathrm{Ni}_{15} \mathrm{HEA}$, whose $\gamma_{\text {isf }}^{\mathrm{DFT}}$ is calculated to be $-26 \mathrm{~mJ} / \mathrm{m}^{2}$, both DT and MT are expected from the nice correlation in Fig. 3, although only the MT was reported from the electron backscatter diffraction (EBSD) results [117], which is likely due to the limited resolution of the EBSD images.

\subsection{Implications of negative SFE}

In the following, we discuss some further implications of the negative SFE. 

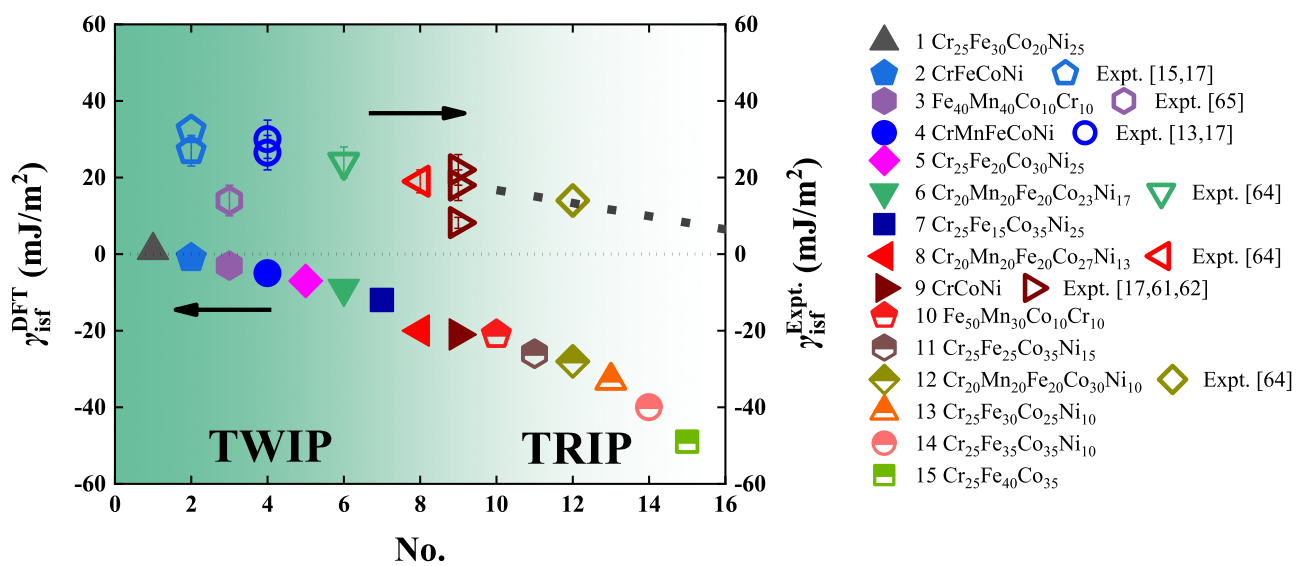

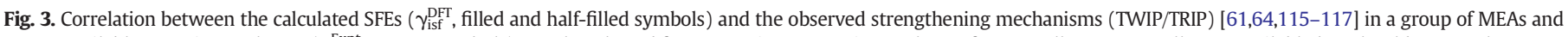

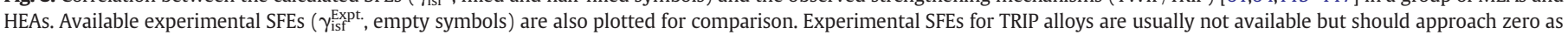
indicated by the dashed line according to the experimental formulas for SFE calculations.

\subsubsection{Interfacial energy $\sigma^{\varepsilon / \gamma}$}

One should be extremely cautious to determine the interfacial energy by subtracting the $\Delta G^{\gamma \rightarrow \varepsilon}$ contribution from the measured SFE $[5,89]$. Since experimentally there is no direct method for measuring the interfacial energy, Olson and Cohen [5] originally proposed that the interfacial energy $\sigma^{\varepsilon / \gamma}$ can be determined via the measured SFE subtracted by the contribution from Gibbs free energy difference $\Delta G^{\gamma \rightarrow \varepsilon}$, i.e., $\sigma^{\varepsilon / \gamma}=\frac{\gamma_{\text {isf }}^{\text {Expt }}}{2}-\frac{\Delta G^{\gamma \rightarrow \varepsilon}}{A}$ (from Eq. (2)). The $\Delta G^{\gamma \rightarrow \varepsilon}$ S of Fe-Cr$\mathrm{Ni}$ alloys were calculated as a function of temperature by using of the regular solution thermodynamics [122]. The $\gamma_{\text {isf }}^{\text {Expt }}$ S of Fe-Cr-Ni alloys were measured by the extended node method $[5,86,123]$ at different temperatures. The interfacial energies $\sigma^{\varepsilon / \gamma}$ S of Fe-Cr-Ni alloys were accordingly determined to be $\sim 10-15 \mathrm{~mJ} / \mathrm{m}^{2}$, which were later on widely adopted in the SFE calculations using the thermodynamic approaches [5]. In light of our above discussions, since the measured SFE decreases toward a small positive value with decreasing $\Delta G^{\gamma \rightarrow \varepsilon}$, the obtained interfacial energy in this way will increase by the similar magnitude as the decrease in the $\Delta G^{\gamma \rightarrow \varepsilon}$ (Fig. 2(c)), as obtained in Ref. [89], which is an artificial effect. The obtained large interfacial energy does not describe the real energetics of the hcp/fcc interface. There are efforts to calculate the composition dependent SFE based on thermodynamic descriptions of $\Delta G^{\gamma \rightarrow \varepsilon}$ for the purpose to design TRIP/TWIP alloys, while the interfacial energy term is commonly treated as a fitting parameter to realize the agreement between the calculated SFE and the $\gamma_{\text {isf }}^{\text {Expt. }}[50,51,89,124]$. In these cases, the $\gamma_{\text {isf }}^{\text {Expt. }}$ values adopted to derive the interfacial energy become critical. A large interfacial energy can effectively wash out the important information carried by $\Delta G^{\gamma \rightarrow \varepsilon}$.

\subsubsection{Effect of negative SFE on the mobility of partial dislocations with re- spect to altering temperature or stress}

Negative SFE is important for understanding the temperature effect on the partial separation width, for the purpose to determine the temperature effect on the SFE $[84,95,125]$. Usually in pure fcc metals and dilute alloys, the temperature induced variation of the SFW is fully reversible. But in concentrated alloys such as in $\mathrm{Ag}-\mathrm{Al}$ [125], $\mathrm{Cu}-\mathrm{Al}$ [95], Co-Ni [84] and Co-Cr-Ni [84] alloys, below a certain temperature $T_{0}$ the SFW does not change with temperature. Above $T_{0}$, partial dislocation can move reversibly as the temperature changes. The irreversible variation in the SFW is usually ascribed to factors like SRO, Suzuki effect, and solute impedance effect, but no consensus has been arrived $[84,95,125]$. However, in accordance with the negative SFE, we can understand the above observations better. For example, in $\mathrm{Cu}-13$ at. $\% \mathrm{Al}$, it was observed that dissociated dislocations generated at room temperature deformation maintain their separation widths unchanged when altering temperature between $77 \mathrm{~K}$ and $450 \mathrm{~K}$ [95]. According to our previous study, this alloy has a negative SFE at room temperature [48] and a positive temperature dependence [95]. Decreasing the temperature from room temperature, the SFE becomes more negative $\left(\sim-15 \mathrm{~mJ} / \mathrm{m}^{2}[48]\right)$, but not smaller enough (together with the repulsive force, Fig. 1(b)) to overcome the lattice friction and push the partials to move, otherwise, thermally induced martensitic phase transformation is expected. When increasing temperature below $T_{0}$, the SFE becomes larger but still negative, there is no driving force to shrink the partial separation because both the SFE and $F_{\text {int }}$ point outwards the partial pair, thus individual partial dislocations appear locked. Only when the SFE is positive and large enough at temperatures higher than $T_{0}$, the SFE and the repulsive force may compete with each other (and with the friction resistance) and alter the partial separation reversibly with respect to temperature as in pure fcc metals. Note that the friction stress should decrease with increasing temperature, which also contributes to the mobility of partials at high temperatures [126,127].

Similarly, the present work renders an alternative explanation for the large variation of the partial separation $d$ of the dislocations with the same character (same $\beta$ ) in HEAs [29]. Smith et al. [29] measured the partial separation of the $a / 2<110>\{111\} 60^{\circ}$ mixed dislocations in $\mathrm{CrMnFeCoNi} \mathrm{HEA} \mathrm{and} \mathrm{found} \mathrm{significantly} \mathrm{large} \mathrm{variations} \mathrm{in} \mathrm{the} \mathrm{dis-}$ sociation distance, compared to the cases of pure fcc metals. It was ascribed to the multicomponent nature of the alloys which have inherent concentration inhomogeneity at nanometer scale in space, thereby, the varying local SFEs $[29,69]$. In fact, in Fe-Mn steels, partial separation was also found to fluctuate dramatically [89]. In a pure fcc metal with positive SFE, there is an equilibrium state of finite partial separation as expected from the force balance between the SFE and the repulsive force (and the small lattice friction resistance) at static conditions. Large deviations in partial separations from the equilibrium distance can possibly be corrected by the SFE or repulsive force driven movements of partials. Therefore, the partial separations only slightly fluctuate around the equilibrium value. While in negative SFE materials, both the SFE and repulsive force tend to separate the partials, competing against the passive lattice friction resistance, thereby, the final separation distances become more sensitive to local shear stresses during deformation (stress history) or the image forces in TEM measurements. One can easily expect that the partials that are separated into different widths by varying local shear stresses should maintain their positions after the external force is removed. Consequently, partial dislocation separations in these alloys display greater variations. 


\section{Conclusions}

We have demonstrated that the extant experimental models for SFE calculations are not valid in metastable alloys, which restrict the obtained $\gamma_{\text {isf }}^{\text {Expt. }}$ always to be positive. Therefore, the $\gamma_{\text {isf }}^{\text {Expt. }}$ values will depart from the true thermodynamic stability of the fcc phase and fail to serve as a proper indicator for the activation of the underlying deformation mechanisms in metastable alloys. On the contrary, the SFEs calculated by DFT methods show nice correlation with the Gibbs energy difference between the fcc and hcp structures in both stable and unstable fcc metals and alloys. We argue that it is of significant importance to embrace the negative SFE for properly understanding dislocation behaviors in metastable systems. Finally, the present work calls for future development of experimental methodologies for measuring the SFE in metastable alloys and a revisit to understand the behaviors of partial dislocations under various deformation conditions in light of the negative SFE.

\section{Data availability}

All data needed to evaluate the conclusions in the paper are present in the paper. Additional data related to this paper may be requested from the authors.

\section{Credit author statement}

Xun Sun: Investigation, Data Curation, Writing-original draft, Visualization Song Lu: Supervision, Conceptualization, Investigation, Writing-reviewing and editing Ruiwen Xie, Xianghai An, Tianlong Zhang, Chunxin Liang, Xiangdong Ding: Data analysis and discussion Wei Li: Software Yunzhi Wang: Discussion, Reviewing Hualei Zhang: Supervision, Writing-reviewing and editing, Funding acquisition Levente Vitios: Supervision, Writing-reviewing and editing, Project administration, Funding acquisition, Resources.

\section{Declaration of Competing Interest}

The authors declare that they have no known competing financial interests or personal relationships that could have appeared to influence the work reported in this paper.

\section{Acknowledgement}

H.Z. acknowledges the financial support from the National Natural Science Foundation of China (No.51871175). X.S. acknowledges the financial support from the China Scholarship Council. X.S., S.L. and L.V. thank the Swedish Research Council (VR, No. 2019-04971), the Swedish Foundation for Strategic Research, the Swedish Foundation for International Cooperation in Research and Higher Education, and the Hungarian Scientific Research Fund (research project OTKA 128229). X.A. acknowledges the financial support from Australia Research Council (DE170100053) and the Robinson Fellowship Scheme of the University of Sydney (G200726). Y. W. acknowledges the support from U.S. National Science Foundation DMREF program under grant DMR1922239. The computations were performed on resources provided by the Swedish National Infrastructure for Computing (SNIC) at the National Supercomputer Centre in Linköping partially funded by the Swedish Research Council through grant agreement no. 2016-07213, and by the "H2" High Performance Cluster at Xi'an, China is acknowledged.

\section{References}

[1] D.J.H. Cockayne, M.L. Jenkins, I.L.F. Ray, The measurement of stacking-fault energies of pure face-centred cubic metals, Philos. Mag. 24 (1971) 1383-1392.
[2] M.J. Mills, P.A. Stadelmann, A study of the structure of lomer and $60^{\circ}$ dislocations in aluminium using high-resolution transmission electron microscopy, Philos. Mag. A 60 (1989) 355-384.

[3] L. Rémy, The interaction between slip and twinning systems and the influence of twinning on the mechanical behavior of fcc metals and alloys, Metall. Trans. A. 12 (1981) 387-408.

[4] O. Grässel, L. Krüger, G. Frommeyer, L.W. Meyer, High strength Fe-Mn-(Al, Si TRIP/TWIP steels development - properties - application, Int. J. Plast. 16 (2000) 1391-1409.

[5] G.B. Olson, M. Cohen, A general mechanism of martensitic nucleation: Part I. General concepts and the FCC $\rightarrow$ HCP transformation, Metall. Trans. A 7 (1976) 1897-1904.

[6] S. Allain, J.P. Chateau, O. Bouaziz, A physical model of the twinning-induced plasticity effect in a high manganese austenitic steel, Mater. Sci. Eng. A 387-389 (2004) 143-147.

[7] H. Idrissi, L. Ryelandt, M. Veron, D. Schryvers, P.J. Jacques, Is there a relationship between the stacking fault character and the activated mode of plasticity of Fe-Mnbased austenitic steels? Scr. Mater. 60 (2009) 941-944.

[8] T.-H. Lee, E. Shin, C.-S. Oh, H.-Y. Ha, S.-J. Kim, Correlation between stacking fault energy and deformation microstructure in high-interstitial-alloyed austenitic steels, Acta Mater. 58 (2010) 3173-3186.

[9] R.M. Latanision, A.W. Ruff, The temperature dependence of stacking fault energy in Fe-Cr-Ni alloys, Metall. Trans. 2 (1971) 505-509.

[10] L. Rémy, A. Pineau, Twinning and strain-induced f.c.c. $\rightarrow$ h.c.p. transformation on the mechanical properties of Co-Ni-Cr-Mo alloys, Mater. Sci. Eng 26 (1976) $123-132$.

[11] B.C. De Cooman, Y. Estrin, S.K. Kim, Twinning-induced plasticity (TWIP) steels, Acta Mater. 142 (2018) 283-362.

[12] K. Lu, L. Lu, S. Suresh, Strengthening materials by engineering coherent internal boundaries at the nanoscale, Science 324 (2009) 349-352.

[13] N.L. Okamoto, S. Fujimoto, Y. Kambara, M. Kawamura, Z.M. Chen, H. Matsunoshita, K. Tanaka, H. Inui, E.P. George, Size effect, critical resolved shear stress, stacking fault energy, and solid solution strengthening in the CrMnFeCoNi high-entropy alloy, Sci. Rep. 6 (2016) 35863.

[14] X.H. An, S.D. Wu, Z.G. Wang, Z.F. Zhang, Significance of stacking fault energy in bulk nanostructured materials: insights from cu and its binary alloys as model systems, Prog. Mater. Sci. 101 (2019) 1-45.

[15] Y. Wang, B. Liu, K. Yan, M. Wang, S. Kabra, Y.-L. Chiu, D. Dye, P.D. Lee, Y. Liu, B. Cai, Probing deformation mechanisms of a FeCoCrNi high-entropy alloy at 293 and 77 K using in situ neutron diffraction, Acta Mater. 154 (2018) 79-89.

[16] X.D. Xu, P. Liu, Z. Tang, A. Hirata, S.X. Song, T.G. Nieh, P.K. Liaw, C.T. Liu, M.W. Chen, Transmission electron microscopy characterization of dislocation structure in a face-centered cubic high-entropy alloy $\mathrm{Al}_{0.1} \mathrm{CoCrFeNi}$, Acta Mater 144 (2018) 107-115.

[17] S.F. Liu, Y. Wu, H.T. Wang, J.Y. He, J.B. Liu, C.X. Chen, X.J. Liu, H. Wang, Z.P. Lu, Stacking fault energy of face-centered-cubic high entropy alloys, Intermetallics 93 (2017) 269-737.

[18] H.Y. Diao, R. Feng, K.A. Dahmen, P.K. Liaw, Fundamental deformation behavior in high-entropy alloys: an overview, Curr. Opin. Solid State Mater. Sci. 21 (2017) 252-266.

[19] J.-W. Yeh, Recent progress in high-entropy alloys, Annales de Chimie Science des Matériaux 31 (2006) 633-648.

[20] A. Gali, E.P. George, Tensile properties of high- and medium-entropy alloys, Intermetallics 39 (2013) 74-78.

[21] T. Ericsson, The temperature and concentration dependence of the stacking fault energy in the co-Ni system, Acta Metall. 14 (1966) 853-865.

[22] P.C.J. Gallagher, The influence of alloying, temperature, and related effects on the stacking fault energy, Metall. Trans. 1 (1970) 2429-2461.

[23] S. Huang, W. Li, S. Lu, F. Tian, J. Shen, E. Holmström, L. Vitos, Temperature dependent stacking fault energy of FeCrCoNiMn high entropy alloy, Scr. Mater. 108 (2015) 44-47.

[24] Z. Dong, S. Schonecker, W. Li, D. Chen, L. Vitos, Thermal spin fluctuations in CoCrFeMnNi high entropy alloy, Sci. Rep. 8 (2018) 12211.

[25] C.K.L. Davies, V. Sagar, R.N. Stevens, The effect of stacking fault energy on the plastic deformation of polycrystalline Ni-Co alloys, Acta Metall. 21 (1973) 1343-1352.

[26] J. Ding, Q. Yu, M. Asta, R.O. Ritchie, Tunable stacking fault energies by tailoring local chemical order in CrCoNi medium-entropy alloys, Proc. Natl. Acad. Sci. U. S. A. 115 (2018) 8919-8924.

[27] S. Zhao, Y. Osetsky, G.M. Stocks, Y. Zhang, Local-environment dependence of stacking fault energies in concentrated solid-solution alloys, Npj Comput. Mater. 5 (2019) 13.

[28] Q.J. Li, H. Sheng, E. Ma, Strengthening in multi-principal element alloys with localchemical-order roughened dislocation pathways, Nat. Commun. 10 (2019) 3563.

[29] T.M. Smith, M.S. Hooshmand, B.D. Esser, F. Otto, D.W. McComb, E.P. George, M. Ghazisaeidi, M.J. Mills, Atomic-scale characterization and modeling of $60^{\circ}$ dislocations in a high-entropy alloy, Acta Mater. 110 (2016) 352-363.

[30] Y. Ikeda, F. Körmann, I. Tanaka, J. Neugebauer, Impact of chemical fluctuations on stacking fault energies of $\mathrm{CrCoNi}$ and $\mathrm{CrMnFeCoNi}$ high entropy alloys from first principles, Entropy 20 (2018) 655

[31] Y. Ikeda, I. Tanaka, J. Neugebauer, F. Körmann, Impact of interstitial C on phase stability and stacking-fault energy of the CrMnFeCoNi high-entropy alloy, Phys. Rev. Mater. 3 (2019) 113603.

[32] A. Rohatgi, K.S. Vecchio, G.T. Gray, The influence of stacking fault energy on the mechanical behavior of $\mathrm{Cu}$ and $\mathrm{Cu}$-Al alloys: deformation twinning, work hardening and dynamic recovery, Metall. Mater. Trans. A 32 (2001) 135-145. 
[33] M. Jo, Y.M. Koo, B.J. Lee, B. Johansson, L. Vitos, S.K. Kwon, Theory for plasticity of face-centered cubic metals, Proc. Natl. Acad. Sci. U. S. A. 111 (2014) 6560-6565.

[34] T.S. Byun, On the stress dependence of partial dislocation separation and deformation microstructure in austenitic stainless steels, Acta Mater. 51 (2003) 3063-3071.

[35] B. Mahato, T. Sahu, S.K. Shee, P. Sahu, T. Sawaguchi, J. Kömi, L.P. Karjalainen, Simultaneous twinning nucleation mechanisms in an Fe-Mn-Si-Al twinning induced plasticity steel, Acta Mater. 132 (2017) 264-275.

[36] N. Naeita, J. Takamura, Deformation twinning in silver-and copper-alloy crystals, Philos. Mag. 29 (1974) 1001-1028.

[37] M.A. Meyers, O. Vöhringer, V.A. Lubarda, The onset of twinning in metals: a constitutive description, Acta Mater. 49 (2001) 4025-4039.

[38] H. Suzuki, C.S. Barrett, Deformation twinning in silver-gold alloys, Acta Metall. 6 (1958) 156-165.

[39] D.R. Steinmetz, T. Jäpel, B. Wietbrock, P. Eisenlohr, I. Gutierrez-Urrutia, A. SaeedAkbari, T. Hickel, F. Roters, D. Raabe, Revealing the strain-hardening behavior of twinning-induced plasticity steels: Theory, simulations, experiments, Acta Mater 61 (2013) 494-510.

[40] I. Gutierrez-Urrutia, S. Zaefferer, D. Raabe, The effect of grain size and grain orientation on deformation twinning in a Fe-22wt.\% Mn-0.6wt.\% C TWIP steel, Mater. Sci. Eng. A 527 (2010) 3552-3560.

[41] J.A. Venables, Deformation twinning in face-centred cubic metals, Philos. Mag. 6 (1961) 379-396.

[42] S. Kibey, J.B. Liu, D.D. Johnson, H. Sehitoglu, Predicting twinning stress in fcc metals: linking twin-energy pathways to twin nucleation, Acta Mater. 55 (2007) 6843-6851.

[43] S.A. Kibey, L.L. Wang, J.B. Liu, H.T. Johnson, H. Sehitoglu, D.D. Johnson, Quantitative prediction of twinning stress in fcc alloys: application to Cu-Al, Phys. Rev. B 79 (2009) 214202

[44] H. Huang, X. Li, Z. Dong, W. Li, S. Huang, D. Meng, X. Lai, T. Liu, S. Zhu, L. Vitos, Critical stress for twinning nucleation in CrCoNi-based medium and high entropy alloys, Acta Mater. 149 (2018) 388-396.

[45] D.J.H. Cockayne, I.L.F. Ray, M.J. Whelan, Investigations of dislocation strain fields using weak beams, Philos. Mag. 20 (1969) 1265-1270.

[46] L.M. Brown, The self-stress of dislocations and the shape of extended nodes, Philos. Mag. 10 (1964) 441-466.

[47] P.M. Anderson, J.P. Hirth, J. Lothe, Theory of Dislocations, Cambridge University Press, New York NY 10006, USA 2017.

[48] W. Li, S. Lu, Q.M. Hu, S.K. Kwon, B. Johansson, L. Vitos, Generalized stacking fault energies of alloys, J. Phys.: Condens. Matter 26 (2014) 265005.

[49] S. Lu, Q.-M. Hu, B. Johansson, L. Vitos, Stacking fault energies of Mn, Co and Nb alloyed austenitic stainless steels, Acta Mater. 59 (2011) 5728-5734.

[50] S. Allain, J.P. Chateau, O. Bouaziz, S. Migot, N. Guelton, Correlations between the calculated stacking fault energy and the plasticity mechanisms in Fe-Mn-C alloys, Mater. Sci. Eng. A 387-389 (2004) 158-162.

[51] S. Curtze, V.T. Kuokkala, A. Oikari, J. Talonen, H. Hänninen, Thermodynamic modeling of the stacking fault energy of austenitic steels, Acta Mater. 59 (2011) 1068-1076.

[52] A. Saeed-Akbari, J. Imlau, U. Prahl, W. Bleck, Derivation and variation in composition-dependent stacking fault energy maps based on subregular solution model in high-manganese steels, Metall. Mater. Trans. A 40 (2009) 3076-3090.

53] X. Li, S. Schönecker, First-principles prediction of the stacking fault energy of gold at finite temperature, Acta Mater. 135 (2017) 88-95.

[54] X. Zhang, B. Grabowski, F. Körmann, A.V. Ruban, Y. Gong, R.C. Reed, T. Hickel, J. Neugebauer, Temperature dependence of the stacking-fault Gibbs energy for $\mathrm{Al}$, Cu, and Ni, Phys. Rev. B 98 (2018) 224106.

[55] Z. Dong, S. Schönecker, D. Chen, W. Li, S. Lu, L. Vitos, Influence of Mn content on the intrinsic energy barriers of paramagnetic FeMn alloys from longitudinal spin fluctuation theory, Int. J. Plast. 119 (2019) 123-139.

[56] X. Sun, H. Zhang, W. Li, X. Ding, Y. Wang, L. Vitos, Generalized stacking fault energy of Al-doped CrMnFeCoNi high-entropy alloy, Nanomaterials 10 (2019) 59.

[57] S. Kibey, J.B. Liu, D.D. Johnson, H. Sehitoglu, Generalized planar fault energies and twinning in Cu-Al alloys, Appl. Phys. Lett. 89 (2006) 191911.

[58] M. Chandran, S.K. Sondhi, First-principle calculation of stacking fault energies in Ni and Ni-Co alloy, J. Appl. Phys. 109 (2011) 103525.

[59] B.E.P. Beeston, I.L. Dillamore, R.E. Smallman, The stacking-fault energy of some nickel-cobalt alloys, Met. Sci. J. 2 (1968) 12-14.

[60] L.-Y. Tian, R. Lizárraga, H. Larsson, E. Holmström, L. Vitos, A first principles study of the stacking fault energies for fcc Co-based binary alloys, Acta Mater. 136 (2017) 215-223.

[61] G. Laplanche, A. Kostka, C. Reinhart, J. Hunfeld, G. Eggeler, E.P. George, Reasons for the superior mechanical properties of medium-entropy $\mathrm{CrCoNi}$ compared to highentropy CrMnFeCoNi, Acta Mater. 128 (2017) 292-303.

[62] R. Zhang, S. Zhao, J. Ding, Y. Chong, T. Jia, C. Ophus, M. Asta, R.O. Ritchie, A.M. Minor, Short-range order and its impact on the CrCoNi medium-entropy alloy, Nature 581 (2020) 283-287.

[63] A.J. Zaddach, C. Niu, C.C. Koch, D.L. Irving, Mechanical properties and stacking fault energies of NiFeCrCoMn high-entropy alloy, JOM 65 (2013) 1780-1789.

[64] S.F. Liu, Y. Wu, H.T. Wang, W.T. Lin, Y.Y. Shang, J.B. Liu, K. An, X.J. Liu, H. Wang, Z.P. Lu, Transformation-reinforced high-entropy alloys with superior mechanical properties via tailoring stacking fault energy, J. Alloys Compd. 792 (2019) 444-455.

[65] S. Picak, J. Liu, C. Hayrettin, W. Nasim, D. Canadinc, K. Xie, Y.I. Chumlyakov, I.V. Kireeva, I. Karaman, Anomalous work hardening behavior of $\mathrm{Fe}_{40} \mathrm{Mn}_{40} \mathrm{Cr}_{10} \mathrm{Co}_{10}$ high entropy alloy single crystals deformed by twinning and slip, Acta Mater. 181 (2019) 555-569.
[66] Z. Zhang, H. Sheng, Z. Wang, B. Gludovatz, Z. Zhang, E.P. George, Q. Yu, S.X. Mao, R.O. Ritchie, Dislocation mechanisms and 3D twin architectures generate exceptional strength-ductility-toughness combination in CrCoNi medium-entropy alloy, Nat. Commun. 8 (2017) 14390.

[67] S. Zhao, G.M. Stocks, Y. Zhang, Stacking fault energies of face-centered cubic concentrated solid solution alloys, Acta Mater. 134 (2017) 334-345.

[68] C. Niu, C.R. LaRosa, J. Miao, M.J. Mills, M. Ghazisaeidi, Magnetically-driven phase transformation strengthening in high entropy alloys, Nat. Commun. 9 (2018) 1363.

[69] Q. Ding, Y. Zhang, X. Chen, X. Fu, D. Chen, S. Chen, L. Gu, F. Wei, H. Bei, Y. Gao, M. Wen, J. Li, Z. Zhang, T. Zhu, R.O. Ritchie, Q. Yu, Tuning element distribution, structure and properties by composition in high-entropy alloys, Nature 574 (2019) 223-227.

[70] S. Lee, M.J. Duarte, M. Feuerbacher, R. Soler, C. Kirchlechner, C.H. Liebscher, S.H. Oh, G. Dehm, Dislocation plasticity in FeCoCrMnNi high-entropy alloy: quantitative insights from in situ transmission electron microscopy deformation, Mater. Res. Lett. 8 (2020) 216-224.

[71] W. Li, S. Lu, Q.-M. Hu, B. Johansson, S.K. Kwon, M. Grehk, J.Y. Johnsson, L. Vitos, Generalized stacking fault energy of $\gamma$-Fe, Philos. Mag. 96 (2016) 524-541.

[72] L. Vitos, Total-energy method based on the exact muffin-tin orbitals theory, Phys. Rev. B 64 (2001), 014107,

[73] L. Vitos, The EMTO Method and Applications, in: Computational Quantum Mechanicals for Materials Engineers, Springer-Verlag, London, 2007.

[74] EMTO, website, http://emto.gitlab.io/ 2018.

[75] J.P. Perdew, K. Burke, M. Ernzerhof, Generalized gradient approximation made simple, Phys. Rev. Lett. 77 (1996) 3865-3868.

[76] B.L. Gyorffy, A.J. Pindor, J. Staunton, G.M. Stocks, H. Winter, A first-principles theory of ferromagnetic phase transitions in metals, J. Phys. F: Met. Phys. 15 (1985) 1337.

[77] H. Zhang, X. Sun, S. Lu, Z. Dong, X. Ding, Y. Wang, L. Vitos, Elastic properties of $\mathrm{Al}_{x} \mathrm{CrMnFeCoNi}(0 \leq x \leq 5)$ high-entropy alloys from $a b$ initio theory, Acta Mater. 155 (2018) 12-22.

[78] Z. Wang, Q. Wu, W. Zhou, F. He, C. Yu, D. Lin, J. Wang, C.T. Liu, Quantitative determination of the lattice constant in high entropy alloys, Scr. Mater. 162 (2019) 468-471.

[79] J.Y. He, W.H. Liu, H. Wang, Y. Wu, X.J. Liu, T.G. Nieh, Z.P. Lu, Effects of Al addition on structural evolution and tensile properties of the FeCoNiCrMn high-entropy alloy system, Acta Mater. 62 (2014) 105-113.

[80] L. Vitos, P.A. Korzhavyi, B. Johansson, Evidence of large magnetostructural effects in austenitic stainless steels, Phys. Rev. Lett. 96 (2006) 117210.

[81] L. Vitos, I.A. Abrikosov, B. Johansson, Anisotropic lattice distortions in random alloys from first-principles theory, Phys. Rev. Lett. 87 (2001) 156401.

[82] D. Ma, B. Grabowski, F. Körmann, J. Neugebauer, D. Raabe, Ab initio thermodynamics of the CoCrFeMnNi high entropy alloy: importance of entropy contributions beyond the configurational one, Acta Mater. 100 (2015) 90-97.

[83] C.B. Carter, I.L.F. Ray, On the stacking-fault energies of copper alloys, Philos. Mag. 35 (1977) 189-200.

[84] L. Rémy, A. Pineau, B. Thomas, Temperature dependence of stacking fault energy in close-packed metals and alloys, Mater. Sci. Eng. 36 (1978) 47-63.

[85] T.C. Tisone, The concentration and temperature dependence of the stacking faul energy in face-centered cubic co-Fe alloys, Acta Metall. 21 (1973) 229-236.

[86] F. Lecroisey, B. Thomas, On the variation of the intrinsic stacking fault energy with temperature in Fe-18 Cr-12 Ni alloys, Phys. Status Solidi A 2 (1970) K217-K220.

[87] L. Remy, Temperature variation of the intrinsic stacking fault energy of a high manganese austenitic steel, Acta Metall. 25 (1977) 173-179.

[88] J.-B. Baudouin, G. Monnet, M. Perez, C. Domain, A. Nomoto, Effect of the applied stress and the friction stress on the dislocation dissociation in face centered cubic metals, Mater. Lett. 97 (2013) 93-96.

[89] D.T. Pierce, J.A. Jiménez, J. Bentley, D. Raabe, C. Oskay, J.E. Wittig, The influence of manganese content on the stacking fault and austenite/ $/ \varepsilon$-martensite interfacial energies in $\mathrm{Fe}-\mathrm{Mn}-(\mathrm{Al}-\mathrm{Si})$ steels investigated by experiment and theory, Acta Mater. 68 (2014) 238-253.

[90] R. Li, S. Lu, D. Kim, S. Schonecker, J. Zhao, S.K. Kwon, L. Vitos, Stacking fault energy of face-centered cubic metals: thermodynamic and $a b$ initio approaches, J. Phys. Condens. Matter 28 (2016) 395001.

[91] A. Howie, P.R. Swann, Direct measurements of stacking-fault energies from observations of dislocation nodes, Philos. Mag. 6 (1961) 1215-1226.

[92] R.P. Reed, R.E. Schramm, Relationship between stacking-fault energy and x-ray measurements of stacking-fault probability and microstrain, J. Appl. Phys. 45 (1974) 4705-4711.

[93] R.E. Schramm, R.P. Reed, Stacking fault energies of seven commercial austenitic stainless steels, Metall. Trans. A. 6 (1975) 1345.

[94] M.L. Jenkins, Measurement of the stacking-fault energy of gold using the weakbeam technique of electron microscopy, Philos. Mag. 26 (1972) 747-751.

[95] H. Saka, Y. Sueki, T. Imura, On the intrinsic temperature dependence of the stacking-fault energy in copper-aluminium alloys, Philos. Mag. A 37 (1978) 273-289.

[96] I.R. Harris, I.L. Dillamore, R.E. Smallman, B.E.P. Beeston, The influence of d-band structure on stacking-fault energy, Philos. Mag. 14 (1966) 325-333.

[97] S. Lu, Q-M. Hu, E.K. Delczeg-Czirjak, B. Johansson, L. Vitos, Determining the minimum grain size in severe plastic deformation process via first-principles calculations, Acta Mater. 60 (2012) 4506-4513.

[98] N. Saunders, M. Fahrmann, M. Fahrmann, THE APPLICATION OF CALPHAD CALCULATIONS TO NI-BASED SUPERALLOYS, in: K.A. Green, T.M. Pollock, R.D. Kissinger (Eds.), Superalloys 2000, TMS, Warrendale 2000, p. 803.

[99] T.L. Achmad, W. Fu, H. Chen, C. Zhang, Z.-G. Yang, Effects of alloying elements concentrations and temperatures on the stacking fault energies of co-based alloys by 
computational thermodynamic approach and first-principles calculations, J. Alloys Compd. 694 (2017) 1265-1279.

[100] I. Shin, E.A. Carter, Possible origin of the discrepancy in Peierls stresses of fcc metals: first-principles simulations of dislocation mobility in aluminum, Phys. Rev. B 88 (2013), 064106, .

[101] Y. Kamimura, K. Edagawa, S. Takeuchi, Experimental evaluation of the Peierls stresses in a variety of crystals and their relation to the crystal structure, Acta Mater. 61 (2013) 294-309.

[102] R. Lizarraga, F. Pan, L. Bergqvist, E. Holmstrom, Z. Gercsi, L. Vitos, First principles theory of the hcp-fcc phase transition in cobalt, Sci. Rep. 7 (2017) 3778.

[103] Z. Wu, H. Bei, G.M. Pharr, E.P. George, Temperature dependence of the mechanical properties of equiatomic solid solution alloys with face-centered cubic crystal structures, Acta Mater. 81 (2014) 428-441.

[104] S. Yoshida, T. Bhattacharjee, Y. Bai, N. Tsuji, Friction stress and hall-Petch relationship in CoCrNi equi-atomic medium entropy alloy processed by severe plastic deformation and subsequent annealing, Scr. Mater. 134 (2017) 33-36.

[105] S. Yoshida, T. Ikeuchi, T. Bhattacharjee, Y. Bai, A. Shibata, N. Tsuji, Effect of elemental combination on friction stress and hall-Petch relationship in face-centered cubic high / medium entropy alloys, Acta Mater. 171 (2019) 201-215.

[106] F.X. Zhang, S. Zhao, K. Jin, H. Xue, G. Velisa, H. Bei, R. Huang, J.Y.P. Ko, D.C. Pagan, J.C. Neuefeind, W.J. Weber, Y. Zhang, Local structure and short-range order in a NiCoCr solid solution alloy, Phys. Rev. Lett. 118 (2017) 205501.

[107] F.X. Zhang, S. Zhao, K. Jin, H. Bei, D. Popov, C. Park, J.C. Neuefeind, W.J. Weber, Y. Zhang, Pressure-induced fcc to hcp phase transition in Ni-based high entropy solid solution alloys, Appl. Phys. Lett. 110 (2017), 011902, .

[108] F. Zhang, Y. Wu, H. Lou, Z. Zeng, V.B. Prakapenka, E. Greenberg, Y. Ren, J. Yan, J.S. Okasinski, X. Liu, Y. Liu, Q. Zeng, Z. Lu, Polymorphism in a high-entropy alloy, Nat. Commun. 8 (2017) 15687.

[109] C.L. Tracy, S. Park, D.R. Rittman, S.J. Zinkle, H. Bei, M. Lang, R.C. Ewing, W.L. Mao, High pressure synthesis of a hexagonal close-packed phase of the high-entropy alloy CrMnFeCoNi, Nat. Commun. 8 (2017) 15634

[110] Y. Chen, Z. Zhou, P. Munroe, Z. Xie, Hierarchical nanostructure of CrCoNi film underlying its remarkable mechanical strength, Appl. Phys. Lett. 113 (2018), 081905,

[111] S. Huang, H. Huang, W. Li, D. Kim, S. Lu, X. Li, E. Holmstrom, S.K. Kwon, L. Vitos, Twinning in metastable high-entropy alloys, Nat. Commun. 9 (2018) 2381.

[112] V.I. Razumovskiy, A. Reyes-Huamantinco, Effect of thermal lattice expansion on the stacking fault energies of fcc Fe and Fe75Mn25 alloy, Phys. Rev. B 93 (2016) 8.

[113] S. Lu, R. Li, K. Kádas, H. Zhang, Y. Tian, S.K. Kwon, K. Kokko, Q.-M. Hu, S. Hertzman, L. Vitos, Stacking fault energy of C-alloyed steels: the effect of magnetism, Acta Mater. 122 (2017) 72-81.
[114] Y. Deng, C.C. Tasan, K.G. Pradeep, H. Springer, A. Kostka, D. Raabe, Design of a twinning-induced plasticity high entropy alloy, Acta Mater. 94 (2015) 124-133.

[115] Z. Li, K.G. Pradeep, Y. Deng, D. Raabe, C.C. Tasan, Metastable high-entropy dualphase alloys overcome the strength-ductility trade-off, Nature 534 (2016) 227-230.

[116] W. Fang, R. Chang, X. Zhang, P. Ji, X. Wang, B. Liu, J. Li, X. He, X. Qu, F. Yin, Effects of cobalt on the structure and mechanical behavior of non-equal molar $\mathrm{Co}_{x} \mathrm{Fe}_{50}$ ${ }_{-x} \mathrm{Cr}_{25} \mathrm{Ni}_{25}$ high entropy alloys, Mater. Sci. Eng. A 723 (2018) 221-228.

[117] W. Fang, R. Chang, P. Ji, X. Zhang, B. Liu, X. Qu, F. Yin, Transformation induced plasticity effects of a non-equal molar Co-Cr-Fe-Ni high entropy alloy system, Metals 8 (2018) 369.

[118] S. Lu, X. Sun, X. An, W. Li, Y. Chen, H. Zhang, L. Vitos, Transformation-mediated twinning, arXiv:1910.11748v1 [cond-mat.mtrl-sci] (2019).

[119] C.E. Slone, S. Chakraborty, J. Miao, E.P. George, M.J. Mills, S.R. Niezgoda, Influence of deformation induced nanoscale twinning and FCC-HCP transformation on hardening and texture development in medium-entropy $\mathrm{CrCoNi}$ alloy, Acta Mater. 158 (2018) 38-52.

[120] J. Miao, C.E. Slone, T.M. Smith, C. Niu, H. Bei, M. Ghazisaeidi, G.M. Pharr, M.J. Mills, The evolution of the deformation substructure in a $\mathrm{Ni}-\mathrm{Co}-\mathrm{Cr}$ equiatomic solid solution alloy, Acta Mater. 132 (2017) 35-48.

[121] W. Guo, Z. Pei, X. Sang, J.D. Poplawsky, S. Bruschi, J. Qu, D. Raabe, H. Bei, Shapepreserving machining produces gradient nanolaminate medium entropy alloys with high strain hardening capability, Acta Mater. 170 (2019) 176-186.

[122] J.F. Breedis, L. Kaufman, The formation of Hcp and Bcc phases in austenitic iron alloys, Metall. Trans. 2 (1971) 2359-2371.

[123] F. Lecroisey, A. Pineau, Martensitic transformations induced by plastic deformation in the Fe-Ni-Cr-C system, Metall. Trans. 3 (1972) 391-400.

[124] J. Nakano, P.J. Jacques, Effects of the thermodynamic parameters of the hcp phase on the stacking fault energy calculations in the Fe-Mn and Fe-Mn-C systems, CALPHAD 34 (2010) 167-175.

[125] H. Saka, T. Kondo, T. Imura, The temperature dependence of the stacking-fault energy in silver-base alloys, Philos. Mag. A 47 (1983) 859-868.

[126] F. Otto, A. Dlouhý, C. Somsen, H. Bei, G. Eggeler, E.P. George, The influences of temperature and microstructure on the tensile properties of a CoCrFeMnNi highentropy alloy, Acta Mater. 61 (2013) 5743-5755.

[127] C. Varvenne, A. Luque, W.A. Curtin, Theory of strengthening in fcc high entropy alloys, Acta Mater. 118 (2016) 164-176. 